\title{
Combination of FTY720 with cisplatin exhibits antagonistic effects in ovarian cancer cells: Role of autophagy
}

\author{
NING ZHANG ${ }^{1,2,3}$, LAN DAI $^{1,2,3}$, YANFEI $^{3}{ }^{3}$, WEN DI $^{1,2}$ and PU XIA ${ }^{3}$ \\ ${ }^{1}$ Department of Obstetrics and Gynecology, Renji Hospital, School of Medicine, Shanghai Jiao Tong University; \\ ${ }^{2}$ Shanghai Key Laboratory of Gynecologic Oncology, Focus Construction Subject of Shanghai Education Department, \\ Key Discipline Project of Renji Hospital, School of Medicine, Shanghai Jiao Tong University, Shanghai, P.R. China; \\ ${ }^{3}$ Signal Transduction Program, Centenary Institute, Sydney Medical School, University of Sydney, Australia
}

Received January 9, 2013; Accepted February 26, 2013

DOI: 10.3892/ijo.2013.1906

\begin{abstract}
Combination therapy with different anticancer drugs has been proven to be an effective strategy for the treatment of various types of cancers, including ovarian cancer. We have previously reported that FTY720 exhibited potent cytotoxic effects in ovarian cancer cells through the necrotic pathway, which differs from the killing effect of cisplatin (CDDP). In the present study, we report that the combination of FTY720 with CDDP yields an unexpected antagonistic effect towards the cytotoxicity of CDDP in a variety of ovarian cancer cell lines, including both CDDP-sensitive and -resistant cells. The antagonistic activity of FTY720 appears ascribable to its effect in autophagy induction. A significant increase in baseline autophagy was observed in CDDP-resistant ovarian cancer cells, compared with the sensitive cells. Blockade of autophagy by either a pharmacological inhibitor (3-MA) or siRNA-mediated knockdown of autophagic gene expression enhances CDDP-induced apoptotic cell death. Notably, by inhibiting autophagy, 3-MA can convert the combination of FTY720 with CDDP from an antagonistic into an additive effect towards killing ovarian cancer cells. Collectively, the findings suggest that a combination of an autophagy regulator with the CDDP-based regime could effectively modulate its efficacy for the treatment of ovarian cancer.
\end{abstract}

\section{Introduction}

Ovarian cancer remains the leading cause of death in women with gynecological malignancies, despite significant

Correspondence to: Professor Pu Xia, Signal Transduction Program, Centenary Institute, Sydney Medical School, University of Sydney, Australia

E-mail: p.xia@centenary.org.au

Professor Wen Di, Department of Obstetrics and Gynecology, Renji Hospital and Institute of Obstetrics and Gynecology, Shanghai Jiao Tong University School of Medicine, Key Laboratory of Gynecologic Cancer Shanghai Municipality, Shanghai, P.R. China

E-mail: diwen163@163.com

Key words: ovarian cancer, cisplatin, combination chemotherapy, FTY720, autophagy improvements in surgical cytoreduction and chemotherapeutic treatment (1). One of the major obstacles to curing ovarian cancer is the cancer exerts a high rate of drug-resistance either inherent or acquired to chemotherapy (2). It is thus imperative to develop more effective therapeutic strategies that circumvent the resistance mechanism of cancer cells.

Autophagy is a process of bulk degeneration of damaged organelles, protein aggregates, and other macromolecules in the cytoplasm (3). It is traditionally regarded as a cellular response to nutrient deprivation or starvation, whereby cells digest a portion of cytoplasm to recycle nutrients for survival $(3,4)$. Previous studies have suggested that autophagy is a general response mechanism to a wide range of cellular stresses $(4,5)$. Autophagy either constitutes stress adaptation machinery for cell survival in certain cellular scenarios, or in other settings, activates an alternative pathway leading to cellular demise. The latter is referred as autophagic cell death (or type II cell death), although the physiological significance of this type of cell death remains controversial $(6,7)$. Notably, radiation and chemotherapeutic drugs that kill cancer cells through apoptosis were often observed to induce autophagy in certain human cancer cells (8). However, the functional relationship between apoptosis and autophagy and the potential cross-regulation between these two processes in cancer chemotherapy are still largely unknown.

FTY720 (2-amino-2-[2-(4-octylphenyl)-1,3-propanediolhydrochloride], also known as Fingolimod, is a synthetic analog of spingosine (9). The drug was originally developed as an immunosuppressive agent (10), which is currently undergoing multiple clinical trials, including prevention of kidney graft rejection (11) and treatment of relapsing multiple sclerosis (12). In addition, FTY720 has also shown preclinical antitumor efficacy in various cancer models, including those of breast, bladder, prostate, lung, liver and hematopoietic malignancies (reviewed in ref. 13). More recently, we have reported that FTY720 exhibited potent cytotoxic effects in ovarian cancer cells through a mechanism differing from that of cisplatin (CDDP), the most commonly used drug in ovarian cancer treatment (14). Combination therapy with different anticancer drugs has been proven to be an effective strategy for overcoming drug resistance and achieving better outcomes in chemotherapy of ovarian cancer. The present study was 
designed to investigate the effect of FTY720 combing with CDDP in ovarian cancer cells. Surprisingly, the combination yields a wide rang of antagonism towards the cytotoxicity of CDDP in a variety of ovarian cancer cell lines, including CDDP-sensitive and -resistant cells. This suggests that combination of FTY720 with CDDP is not an ideal combinational regime, although they exert their own anticancer efficacy through distinct killing mechanisms.

\section{Materials and methods}

Cell lines and reagents. Human ovarian carcinoma cell lines A2780, 2008, SKOV-3 and IGROV-1 were cultured in DMEM medium supplemented with $10 \%$ heat-inactivated fetal calf serum, penicillin $(100 \mathrm{U} / \mathrm{ml})$ and streptomycin $(100 \mu \mathrm{g} / \mathrm{ml})$ at $37^{\circ} \mathrm{C}$ in a humidified atmosphere of $5 \% \mathrm{CO}_{2}$. FTY720 and SKI-II (4-[4-(chlorophenyl)-2-thiazolyl]aminophenol) were purchased from Cayman (Ann Arbor, MI, USA). Cis-diaminedichloroplatinum (CDDP), 3-methyladenine (3-MA) and all other chemicals were purchased from SigmaAldrich (St. Louis, MO, USA).

Cell viability assay. Cells (3,000 cell/well) were seeded in three to four replication into 96-well plates and incubated with or without FTY720 at the indicated concentration for a desired time period. After the indicated treatments, cell viability was assessed using the MTS (3-(4, 5-dimethylthiazol-2-yl)5-(3-carboxymethoxyphenyl)-2-(4-sulfophenyl)-2H-tetrazolium, inner salt; Sigma) assay as described previously (15). The absorbance intensity of the MTS product is directly proportional to the number of viable cells in culture when cell number is between 2,000 and 200,000; otherwise the exponential dependence was determined. The cell viability was expressed as a percentage of absorbance in cells with indicated treatments to that in cells with vehicle control treatment.

Clonogenic assay. Cells were treated for $24 \mathrm{~h}$ with the indicated drugs, washed and incubated in drug-free media for 14 days. Cells were fixed with $70 \%$ methanol and stained with crystal violet. Colonies, defined as clusters of $\geq 50$ cells, were scored manually with the aid of a Nikon TS100 inverted microscope. Clonogenic survival was expressed as the percentage of colonies formed in drug-treated wells with respect to vehicle alone. Under standard conditions, $>100$ colonies were formed in untreated control cells following 14 days of incubation.

Combined drug effect analysis. The multiple drug effect analysis of Chou and Talalay was used to evaluate combined drug effects, which is based on the median-effect principle (16). The combination index (CI) and dose-reduction index (DRI) were calculated for determining the mode of interaction (synergism, antagonism and additive effect) between FTY720 and CDDP as described by Chou and Talalay $(16,17)$. CompuSyn software was applied for above drug combination analysis as described previously (18).

Immunoblot analysis. Equal amounts of proteins collected from cell lysates were resolved by $8-15 \%$ SDS-PAGE using a NuPAGE system (Invitrogen, Carlsbad, CA, USA) and then transferred onto PVDF membranes according to the manu- facturer's instructions. After blocking with 5\% non-fat milk in PBS containing 0.1\% Tween-20, the PVDF membranes were probed with primary antibodies against Beclin-1, LC-3 (Novus Biologicals, Littleton, CO, USA) and $\beta$-actin (Sigma) at $4^{\circ} \mathrm{C}$ overnight, followed by horseradish peroxidaseconjugated proper second antibodies. The immunoreactivities were detected with enhanced chemiluminescence Plus kit (GE Healthcare, Piscataway, NJ, USA). Densitometry was performed on a Kodak Imager.

Fluorescence microscope. The subcellular localization of LC3 was examined in cells overexpressing GFP-LC3 (gift from Dr M.J. Zhao, Shanghai Institutes for Biological Science, China) by fluorescence microscopic analysis. Cells displaying a diffuse distribution of GFP-LC3 in the cytoplasm and nucleus were considered non-autophagic, whereas cells presenting intense GFP-LC3 punctations with no nuclear GFP-LC were classified as autophagic formation. Autophagy was quantified by counting the percentage of GFP-LC3-transfected cells showing accumulation of GFP-LC3 in puncta ( $\geq 100$ cells were counted for each experiment).

RNA interference. Chemically synthesized scrambled RNAi oligonucleotides and siRNA targeting human Beclin 1 and LC3 were purchased from GenePharma (Shanghai, China). The siRNA corresponding to human cDNA sequence are: Beclin 1, GAA TGT CAG AAC TAC AAA CGC TGT T; LC3, GAA GGC GCU UAC AGC UCA A. The siRNA transfection was performed using Hiperfect reagent (Qiagen, Germantown, MD, USA) according to the manufacturer's protocol. The expression levels of targeted genes were detected by western blot assays after 36-48-h transfection.

Statistical analysis. SPSS11.0 software package was used to perform statistical analysis. Unpaired Student's t-tests were used for comparison between two groups. For multiple comparisons, results were analyzed by ANOVA followed by the Dunnet's test. A $\mathrm{p}<0.05$ was considered statistically significant.

\section{Results}

FTY720-induced cytotoxicity in ovarian cancer cells is cell density-dependent. Our previous study reported that FTY720 exhibits a potent cytotoxic effect in ovarian cancer cells via non-apoptotic programmed cell death pathways, which is distinct from the CDDP-mediated apoptotic cell death (14). In an attempt to further characterize the killing mechanism of FTY720, we investigated the influence of cell density on the cytotoxicity of FTY720 in ovarian cancer cells. Consistent with our previous report, FTY720 induced cell death in subconfluent cultures in a dose-dependent manner in ovarian cancer cells (Fig. 1). However, the sensitivity to FTY720 was significantly reduced when the treatment was conducted on cells reaching confluence. Moreover, almost no cell death was observed in over-confluent cultures treated with FTY720 (Fig. 1). In contrast, CDDP induced cell death was independent of the cell density, irrespective of cellular sensitivity to CDDP (Fig. 1A). These observations further strengthen the notion that FTY720 functions through a distinct pathway to CDDP in killing of ovarian cancer cells. 

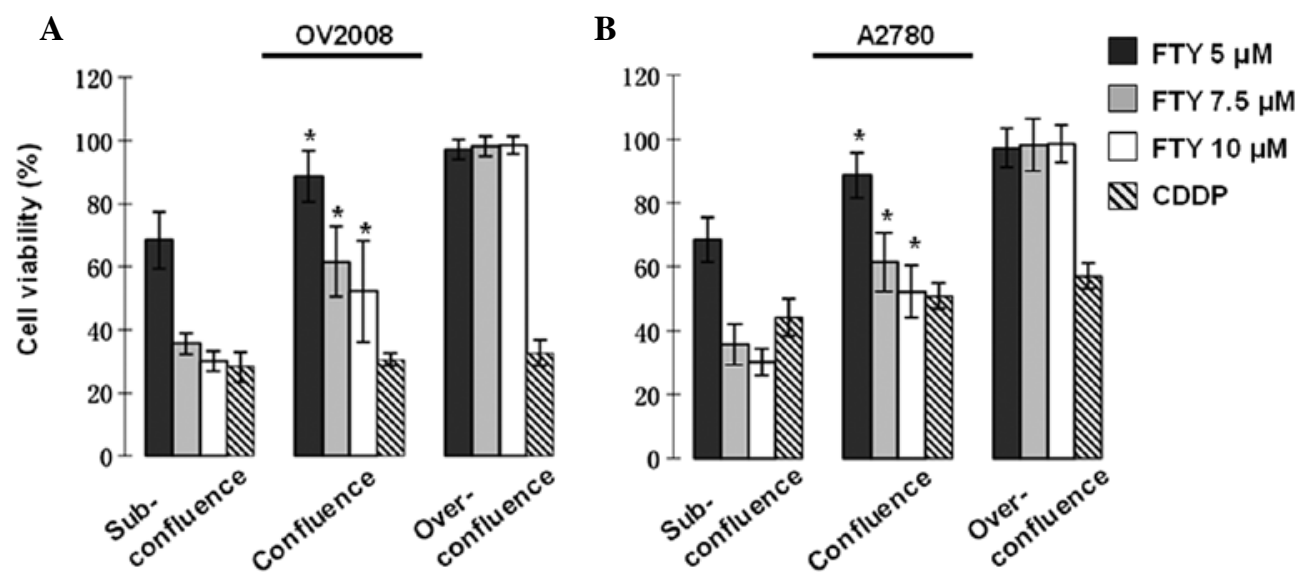

Figure 1. FTY720 induces cytotoxicity in ovarian cancer cells dependent on cell density. OV2008 (A) and A2780 (B) ovarian cancer cell lines were plated with different cell densities and treated with various doses of FTY720 (5, 7.5 and $10 \mu \mathrm{M})$ or CDDP $(10 \mu \mathrm{M})$. Cell viability was then determined post 12 -h treatment. Data are expressed as the percentage (\%) of control group without treatment and represented means \pm SD from three independent experiments. $\mathrm{p}<0.05$, confluent cultures compared with sub-confluent or over-confluent cultures.
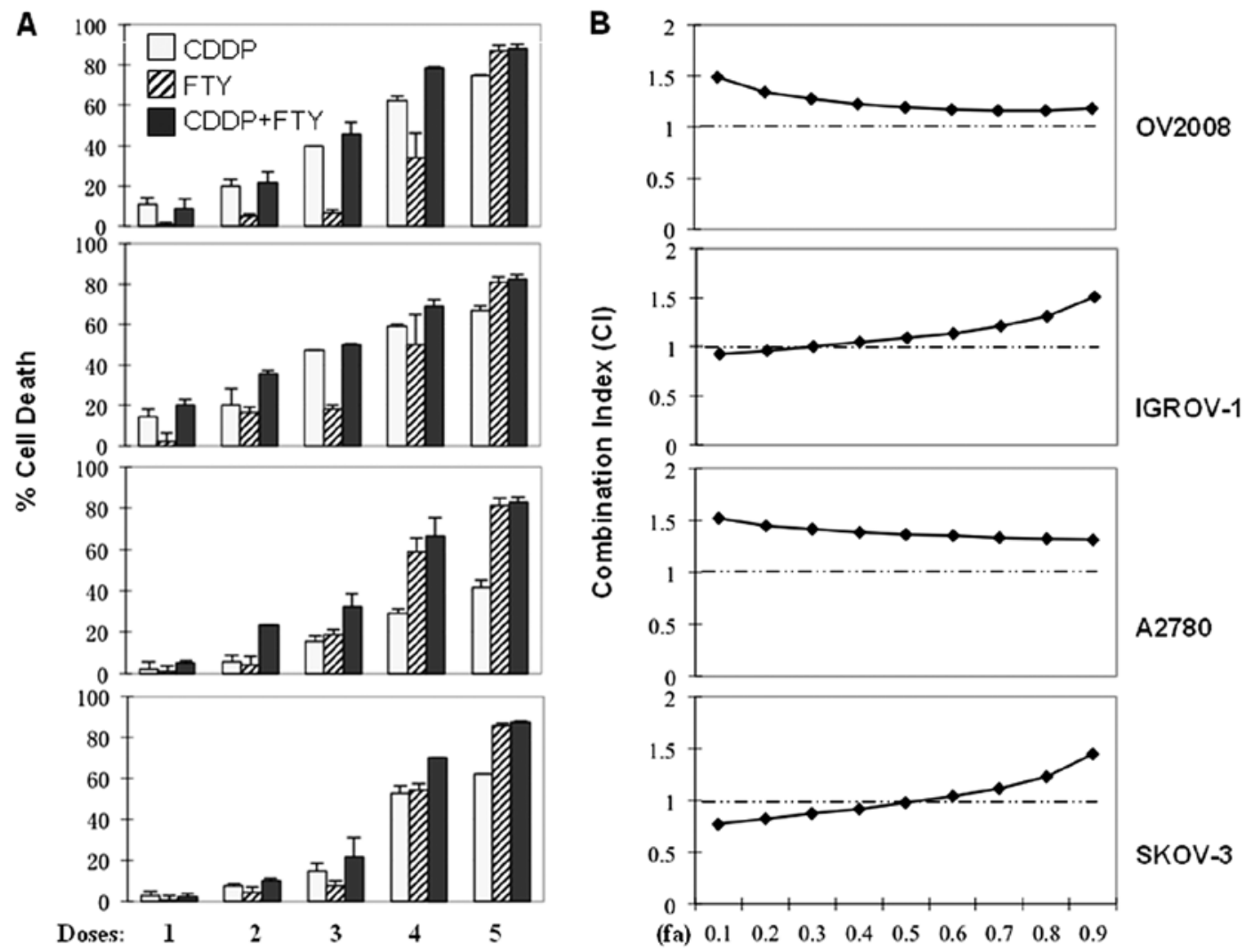

Figure 2. Combined effect of FTY720 with CDDP in ovarian cancer cells. (A) OV2008, IGROV-1, A2780 and SKOV-3 cells were cultured reaching confluence and treated for $24 \mathrm{~h}$ with increasing concentrations of $\operatorname{CDDP}(2.5,5.0,10,20$ and $40 \mu \mathrm{M}$, respectively for D1-D5) and/or FTY720 (1.25, 2.5, 5, 10 and $20 \mu \mathrm{M}$, respectively for D1-D5). The percentages of cell death were measured by MTS assay. All values represent the means with bars as SD of three samples in an independent experiment, which were repeated three times with similar results. (B) Combination index (CI) of CDDP and FTY720 on various ovarian cancer cells was calculated as described $(16,17)$. Here fa in X-axis denotes fraction affected (e.g., fa of 0.5 is equivalent to a $50 \%$ of cell death).

Combination of FTY720 with CDDP exhibits an antagonistic effect on the cytotoxicity in ovarian cancer cells. Combination of CDDP with other therapeutic agents that exert different killing mechanisms has becoming the predominate trend for better clinical outcomes of platinum-based chemotherapy in ovarian cancer patients $(19,20)$. Thus, we evaluated the effect of FTY720 in combination with CDDP in both CDDP-sensitive
(OV2008 and IGROV-1) and -resistant (A2780 and SKOV-3) ovarian cancer cells. As reported previously (14), treatment with either FTY720 or CDDP resulted in a dose-dependent cytotoxicity in these ovarian cancer cell lines, although A2780 and SKOV-3 cells exhibited resistance to CDDP to a certain degree (Fig. 2A). However, combination of FTY720 and CDDP did not yield any additive cytotoxic effects on these 
A


Figure 3. Difference in autophagy levels between CDDP-sensitive and refractory cells. (A) OV2008, SKOV-3, A2780 and IGROV-1 cells were treated with vehicle or indicated concentration of CDDP for $24 \mathrm{~h}$. LC3-II formation was then determined by immunoblot analysis. Similar results were obtained in three independent experiments. (B) OV2008 or A2780 cells were transiently transfected with enhanced green fluorescent protein-labeled LC3 (pEGFP-LC3) and visualized by fluorescence microscopy post 24-h transfection. The representative images of GFP-LC3 staining are shown (left panels) and the percentage of GFP-positive cells presenting typical GFP-LC3 puncta ( $>5$ puncta per cell) is depicted (right panel). Data are means \pm SD of three independent experiments $(\geq 100$ cells were counted in each experiment).

ovarian cancer cells (Fig. 2A). For detailed analysis of the combinational drug effect, we determined the mass-action law parameters, including $\mathrm{Dm}, \mathrm{m}$ and $\mathrm{r}$ values, which represent the potency, shape of dose-effect curve and the conformity to the mass-action law, respectively, for each drug and their combinations (Table I). These Dm and $\mathrm{m}$ values were then used to calculate the combination index $(\mathrm{CI})$ values based on the Chou-Talalay method, where CI $<1,=1$ and $>1$, indicate synergism, additive effect and antagonism, respectively $(16,17)$. The CI-fa (fraction affected) curves of FTY720/CDDP combination show the $\mathrm{CI}$ values ranging from 0.97 to 1.51 at different effect levels from $\mathrm{IC}_{50}$ to $\mathrm{IC}_{90}$ (Fig. 2B), indicating an extensive range of antagonism in the combinational treatment.

CDDP-resistant cells exhibit increased levels of autophagy. We have previously reported that in addition to its cytotoxic effect, FTY720 induces autophagy in ovarian cancer cells (14). We thought that the antagonistic effect of FTY720 in the combination with CDDP might be attributed to autophagy formation. To test this hypothesis, we firstly investigated the potential role of autophagy in ovarian cancer cells response to CDDP. Interestingly, the CDDP-resistant cells, SKOV3 and A2780, exhibited a significant increase in baseline levels of autophagy, as determined by LC3 expression and its lipidation status (LC3-II), compared with the sensitive OV2008 and IGROV-1 cells (Fig. 3A). The increased levels of autophagy in the resistant cells remained following CDDP treatment (Fig. 3A). To further confirm the difference in autophagy levels between CDDP-resistant and sensitive cells, we trans-
Table I. Dose-effect relationship parameters of FTY720 or/and CDDP against various ovarian cancer cell lines.

\begin{tabular}{llcccc}
\hline Cell lines & Compound & $\mathrm{m}$ & $\operatorname{Dm}(\mu \mathrm{M})$ & $\mathrm{r}$ & $\mathrm{n}$ \\
\hline OV2008 & FTY720 & 2.416 & 11.15 & 0.912 & 5 \\
& CDDP & 1.193 & 14.83 & 0.988 & 5 \\
& FTY720+CDDP & 1.627 & 16.02 & 0.984 & 5 \\
IGROV-1 & FTY720 & 1.555 & 9.26 & 0.917 & 5 \\
& CDDP & 0.980 & 15.74 & 0.907 & 5 \\
& FTY720+CDDP & 0.995 & 13.86 & 0.995 & 5 \\
A2780 & FTY720 & 2.486 & 10.10 & 0.951 & 5 \\
& CDDP & 1.564 & 25.21 & 0.940 & 5 \\
& FTY720+CDDP & 2.103 & 22.98 & 0.976 & 5 \\
SKOV-3 & FTY720 & 2.244 & 9.63 & 0.990 & 5 \\
& CDDP & 1.164 & 48.10 & 0.979 & 5 \\
& FTY720+CDDP & 1.405 & 20.07 & 0.966 & 5
\end{tabular}

The parameters $\mathrm{m}, \mathrm{Dm}$ and $\mathrm{r}$ signify the shape of the dose-effect curve, the potency $\left(\mathrm{IC}_{50}\right)$ and conformity of the data to the mass-action law. $n$, is the number of sets of dose-effect relationship experiments that were carried out. $\mathrm{m}$ and Dm values were calculated as described $(16,17)$.

fected GFP-LC3 into A2780 and OV2008 cells and monitored the accumulation of GFP-LC3 puncta in the transfected cells. As seen in Fig. 3B, the resistant A2780 cells showed a signifi- 
A
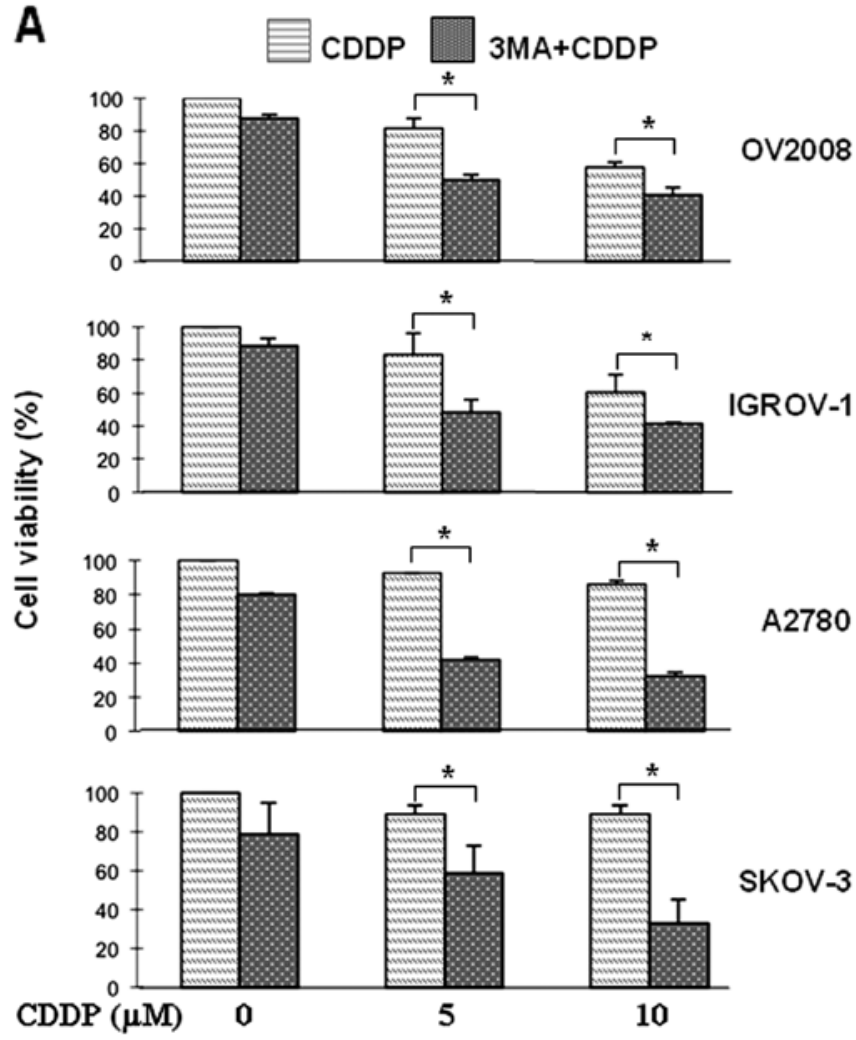

B
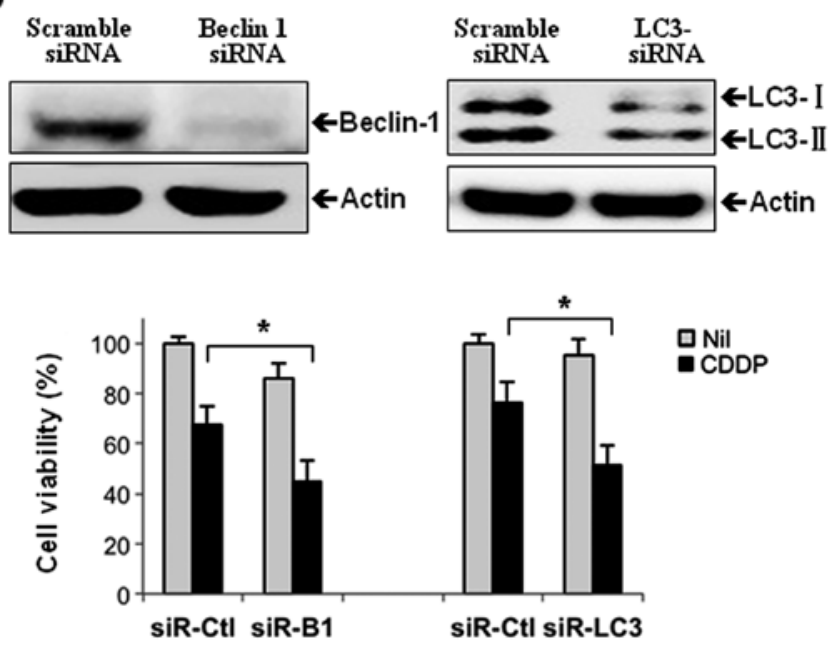

Figure 4. Effect of autophagy inhibition on the sensitivity of ovarian cancer cells to CDDP treatment. (A) OV2008, IGROV-1, A2780 and SKOV-3 cells were treated for $24 \mathrm{~h}$ with increasing concentrations of CDDP $(0,5$ and $10 \mu \mathrm{M})$ in the absence or presence of 3-MA $(5 \mathrm{mM})$ and then the cell viability was measured by MTS assays. (B) A2780 cells were transfected with specific siRNAs targeting human Beclin 1 or LC-3 and scrambled control siRNA for $48 \mathrm{~h}$ and expression levels of Beclin 1 LC3-I and LC3-II were determined by immunoblot assays (top panels). Cell viability (bottom panels) was determined in the siRNA-transfected cells exposed to CDDP $(10 \mu \mathrm{M})$ for an additional $24 \mathrm{~h}$. Data are means \pm SD from three independent experiments $\left({ }^{*} \mathrm{p}<0.01\right)$.

cant increase in the number of cells with puncta fluorescence, whereas the majority of GFP-LC3 transfected OV2008 cells displayed a diffused distribution of fluorescence. Together, these findings indicated that CDDP-resistant ovarian cancer cells exhibit elevated levels of autophagy compared to CDDPsensitive cells.
Blockage of autophagy augments CDDP-induced cell death. To examine the role of autophagy in the cytotoxic effect of CDDP in ovarian cancer cells, we employed the pharmacological inhibitor, 3-methyladenine (3-MA), that has been demonstrated to effectively block the formation of autophagosome (21). Notably, treatment with 3-MA resulted in a significant increase in CDDP-induced cell death in either CDDP-sensitive or -resistant cells (Fig. 4A). In order to exclude off-target effects of the chemical inhibitor 3-MA, we used siRNA to specifically knock-down the expression of LC3 and Beclin 1, known to be crucial for autophagy formation and function (22). As shown in Fig. 4B, transfection of A2780 cells with either Beclin 1- or LC3-siRNA significantly decreased the target protein expression, compared with the control siRNA-transfected cells. In line with the 3-MA experimental data, silencing of Beclin 1 or LC3 expression significantly enhanced cellular response to CDDP killing effect (Fig. 4B, bottom panels). Furthermore, inhibition of autophagy by 3-MA reverses the antagonism of FTY720 into a significant additive killing effect in its combination with CDDP in either the CDDP-sensitive OV2008 cells or the resistant A2780 cells (Fig. 5A). This observation was further confirmed by long-term colony formation assays (Fig. 5B). Collectively, these data suggest that autophagy is capable of affecting cell response to CDDP treatment and that blockade of autophagy renders ovarian cancer cells susceptible to the cytotoxicity of either CDDP or FTY720 or their combination.

\section{Discussion}

Drug resistance is still a major obstacle to successful treatment of ovarian cancer. While platinum-based chemotherapy has achieved a high response rate, the great majority relapses and develops resistance to further chemotherapy $(19,20)$. As such, CDDP combination therapy with multiple drugs or multiple modalities that overcome drug resistance is one of the major strategies for achieving better therapeutic outcomes of ovarian cancer (2).

FTY720, a synthetic analog of sphingosine, has been shown to have a powerful therapeutic potential in the treatment of various types of cancers (13). In our previous studies, we found that FTY720 exerts a potent cytotoxic effect against ovarian cancer cells via the apoptosis-independent programmed cell death pathway accompanied with autophagy formation (14). The FTY720-induced cytotoxicity is also demonstrated to be clearly distinct from CDDP-mediated apoptotic cell death in ovarian cancer cells (14). Such a difference has been further demonstrated by the present study, showing that FTY720induced cell death is cell density-dependent, whereas CDDP killing effect is independent of cell density (Fig. 1). We expected that the distinct killing mechanisms of FTY720 and CDDP might lead to a potent and effective combination of these two agents for treatment of ovarian cancer cells. Surprisingly, the combination index analysis revealed that FTY720/CDDP combination exhibited a broad rang of antagonism in both CDDP-sensitive (OV2008 and IGROV-1) and -resistant (A2780 and SKOV-3) ovarian cancer cells, with CI ranging from 0.97 to 1.51 for $\mathrm{IC}_{50}$ to $\mathrm{IC}_{90}$. This suggests that the combination of FTY720 with CDDP does not produce a favorable interaction for their cytotoxicity in ovarian cancer cells in vitro or in vivo. 
A

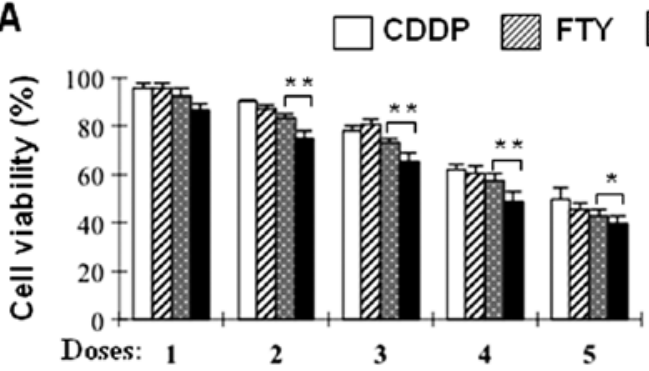

B
OV2008
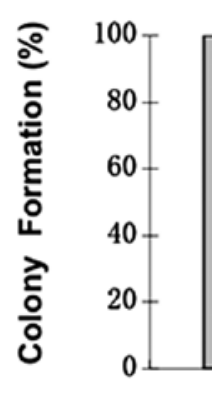

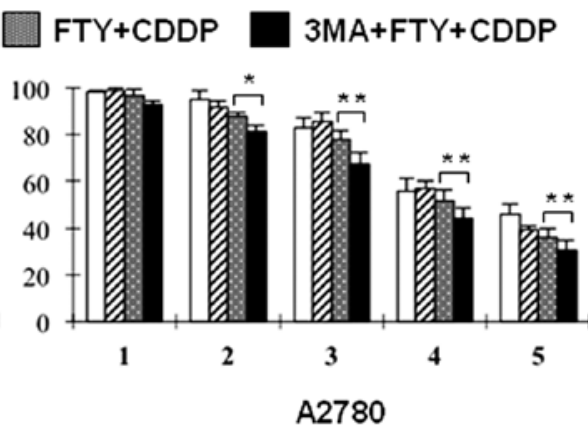

A2780

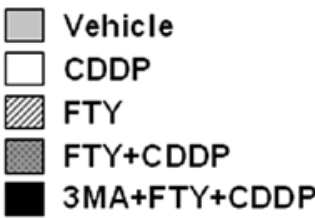

3MA+FTY+CDDP

Figure 5. Autophagy inhibition modulates the combined effect of FTY720 with CDDP in ovarian cancer cells. (A) OV2008 and A2780 cells were exposed to increasing concentrations of CDDP $(2.5,5.0,10,20$ and $40 \mu \mathrm{M}$, respectively for D1-D5) and/or FTY720 (1.25, 2.5, 5, 10 and 20 $\mu \mathrm{M}$, respectively for D1-D5) for $24 \mathrm{~h}$ in the absence or presence of 3-MA $(5 \mathrm{mM})$ and then cell viability was determined. (B) Clonogenic survival assays were conducted in OV2008 and A2780 cells treated for $24 \mathrm{~h}$ with vehicle, CDDP $(5 \mu \mathrm{M})$, FTY720 $(2.5 \mu \mathrm{M})$ or combination of CDDP and FTY720 in the absence or presence of $3-\mathrm{MA}(2.5 \mathrm{mM})$ as described in Materals and methods. Data are means \pm SD of triplicates. ${ }^{*} \mathrm{p}<0.05,{ }^{* *} \mathrm{p}<0.01$; presence vs absence of 3-MA.

Instead, the combination of FTY720 and CDDP results in an antagonistic effect, although these two individual drugs induce cell death through strikingly different mechanisms.

In an attempt to investigate the possible mechanisms by which FTY720 antagonizes the cytotoxicity of CDDP, we examined the role of autophagy, as it has been suggested to be an important protective mechanism against anticancer therapies (8). Indeed, the ability of FTY720 to induce autophagy formation and enhance autophagic flux in ovarian cancer cells has been demonstrated by our earlier studies (14). Therefore, we thought that the antagonism resulted from the combination of FTY720 with CDDP may be ascribable to the protective role of autophagy in refractory response to CDDP treatment. This notion is supported by our findings. The CDDP-resistant SKOV3 and A2780 cells exhibit a significantly higher level of autophagy than that in the sensitive OV2008 and IGROV-1 cells under both basal and drug-stimulated conditions, as determined by the expression levels of LC3-II and GFP-fussed LC3 translocation (Fig. 3). Notably, inhibition of autophagy by using small-molecule inhibitor 3-MA strengthened the cytotoxicity of CDDP in all ovarian cancer cells that we tested. The sensitizing effects of 3-MA appear independent of cellular responsiveness to CDDP (Fig. 4). Blockage of autophagy formation by the siRNA-mediated knockdown of LC3 and Beclin 1 expression significantly enhanced the killing effect of CDDP. Moreover, inhibition of autophagy by 3-MA converted the combination of FTY720 with CDDP from an antagonistic into an additive effect towards to killing ovarian cancer cells (Fig. 5). These observations not only confirm the effect of FTY720 in autophagy induction, but also suggest that autophagy is, at least partially, responsible for the antagonistic of FTY720 in its combination with CDDP. Interestingly, a recent study showed that combination of FTY720 with milatuzumab, an anti-CD74 mAb, resulted in a significant synergistic effect in treatment of mantle cell lymphoma cells (23). The synergistic effect of FTY720 was suggested to be ascribable to its function in blockage of autophagic flux and disruption of the autophagic-lysosomal degradation of CD74 (23). The inhibitory effect of FTY720 on autophagy in the lymphoma cells strikingly conflicts with the findings that FTY720 induces autophagy formation and promotes autophagic flux in ovarian cancer cells and other neoplastic cells, as reported from our studies and others (14,24-26). This discrepancy may suggest that the effect of FTY720 on autophagy is cell type-dependent. Notably, there is accumulating evidence showing autophagy can function as either a pro-survival or a pro-death mechanism in a highly context-dependent manner (6). As such, the actions of FTY720 on autophagy should be taken into account in evaluation of the anticancer efficacy of FTY720, especially in its combination with other chemotherapeutic agents.

There is considerable evidence indicating that autophagy is often induced in response to various stress conditions, such as exposure to anticancer drugs or other cytotoxic agents (8,27-29). Under these conditions, autophagy has been suggested to play an important cytoprotective role for cell survival $(3,29,30)$. Indeed, inhibition of autophagy has been shown to enhance the anticancer efficacy of various anticancer therapies $(8,31,32)$. Along with these observations, our findings further support the concept that inhibition of autophagy may represent a promising strategy for the treatment of refractory cancers, including ovarian cancer. 


\section{Acknowledgements}

We are grateful to Dr M.J. Zhao for providing GFP-LC3 constructs. This study was supported by grants from Australian National Health and Medical Research Council (Program 571408; P.X.) and Chinese National Natural Science Foundation (81072137, W.D.), National Natural Science Foundation (81101972,N.Z.) and Science and Technology Fund of Shanghai Jiaotong University School of Medicine (11XJ21018, N.Z.), Ph.D. Programs Foundation of Ministry of Education of China (20100073110075, W.D.) and Shanghai Education Commission Key Disciplines Foundation. P.X. is supported by Australian Cancer Institute NSW Research Fellowship.

\section{References}

1. Jemal A, Siegel R, Ward E, Hao Y, Xu J and Thun MJ: Cancer statistics, 2009. CA Cancer J Clin 59: 225-249, 2009.

2. Bast RC Jr, Hennessy B and Mills GB: The biology of ovarian cancer: new opportunities for translation. Nat Rev Cancer 9: 415-428, 2009.

3. Shintani T and Klionsky DJ: Autophagy in health and disease: a double-edged sword. Science 306: 990-995, 2004.

4. Levine B and Kroemer G: Autophagy in the pathogenesis of disease. Cell 132: 27-42, 2008.

5. Mizushima N, Levine B, Cuervo AM and Klionsky DJ: Autophagy fights disease through cellular self-digestion. Nature 451: 1069-1075, 2008.

6. Maiuri MC, Zalckvar E, Kimchi A and Kroemer G: Self-eating and self-killing: crosstalk between autophagy and apoptosis. Nat Rev Mol Cell Biol 8: 741-752, 2007.

7. Kroemer $\mathrm{G}$ and Levine B: Autophagic cell death: the story of a misnomer. Nat Rev Mol Cell Biol 9: 1004-1010, 2008.

8. Kondo Y, Kanzawa T, Sawaya R and Kondo S: The role of autophagy in cancer development and response to therapy. Nat Rev Cancer 5: 726-734, 2005.

9. Suzuki S, Enosawa S, Kakefuda T, Shinomiya T, Amari M, Naoe S, Hoshino Y and Chiba K: A novel immunosuppressant, FTY720, with a unique mechanism of action, induces long-term graft acceptance in rat and dog allotransplantation. Transplantation 61: 200-205, 1996.

10. Suzuki S, Enosawa S, Kakefuda T, Li XK, Mitsusada M, Takahara S and Amemiya H: Immunosuppressive effect of a new drug, FTY720, on lymphocyte responses in vitro and cardiac allograft survival in rats. Transpl Immunol 4: 252-455, 1996.

11. Tedesco-Silva H, Mourad G, Kahan BD, Boira JG, Weimar W, Mulgaonkar S, Nashan B, Madsen S, Charpentier B, Pellet P and Vanrenterghem Y: FTY720, a novel immunomodulator: efficacy and safety results from the first phase $2 \mathrm{~A}$ study in de novo renal transplantation. Transplantation 77: 1826-1833, 2004.

12. Pelletier D and Hafler DA: Fingolimod for multiple sclerosis. N Engl J Med 366: 339-347, 2012.

13. Vadas M, Xia P, McCaughan G and Gamble J: The role of sphingosine kinase 1 in cancer: oncogene or non-oncogene addiction? Biochim Biophys Acta 1781: 442-447, 2008.

14. Zhang N, Qi Y, Wadham C, Wang L, Warren A, Di W and Xia P: FTY720 induces necrotic cell death and autophagy in ovarian cancer cells: a protective role of autophagy. Autophagy 6: $1157-1167,2010$

15. Sukocheva O, Wang L, Verrier E, Vadas MA and Xia P: Restoring endocrine response in breast cancer cells by inhibition of the sphingosine kinase-1 signaling pathway. Endocrinology 150: 4484-4492, 2009.
16. Chou TC and Talalay P: Quantitative analysis of dose-effect relationships: the combined effects of multiple drugs or enzyme inhibitors. Adv Enzyme Regul 22: 27-55, 1984.

17. Chou TC: Theoretical basis, experimental design, and computerized simulation of synergism and antagonism in drug combination studies. Pharmacol Rev 58: 621-681, 2006.

18. Zhang N, Wu ZM, McGowan E, Shi J, Hong ZB, Ding CW, Xia P and Di W: Arsenic trioxide and cisplatin synergism increase cytotoxicity in human ovarian cancer cells: therapeutic potential for ovarian cancer. Cancer Sci 100: 2459-2464, 2009.

19. Agarwal R and Kaye SB: Ovarian cancer: strategies for overcoming resistance to chemotherapy. Nat Rev Cancer 3: 502-516, 2003.

20. Muggia F: Platinum compounds 30 years after the introduction of cisplatin: implications for the treatment of ovarian cancer. Gynecol Oncol 112: 275-281, 2009.

21. Seglen PO and Gordon PB: 3-Methyladenine: specific inhibitor of autophagic/lysosomal protein degradation in isolated rat hepatocytes. Proc Natl Acad Sci USA 79: 1889-1892, 1982.

22. Tassa A, Roux MP, Attaix D and Bechet DM: Class III phosphoinositide 3-kinase - Beclin1 complex mediates the amino acid-dependent regulation of autophagy in $\mathrm{C} 2 \mathrm{C} 12$ myotubes. Biochem J 376: 577-586, 2003.

23. Alinari L, Mahoney E, Patton J, Zhang X, Huynh L, Earl CT, Mani R, Mao Y, Yu B, Quinion C, Towns WH, Chen CS, Goldenberg DM, Blum KA, Byrd JC, Muthusamy N, Praetorius-Ibba M and Baiocchi RA: FTY720 increases CD74 expression and sensitizes mantle cell lymphoma cells to milatuzumab-mediated cell death. Blood 118: 6893-6903, 2011.

24. Romero Rosales K, Singh G, Wu K, Chen J, Janes MR, Lilly MB, Peralta ER, Siskind LJ, Bennett MJ, Fruman DA and Edinger AL: Sphingolipid-based drugs selectively kill cancer cells by down-regulating nutrient transporter proteins. Biochem J 439: 299-311, 2011.

25. Wallington-Beddoe CT, Hewson J, Bradstock KF and Bendall LJ: FTY720 produces caspase-independent cell death of acute lymphoblastic leukemia cells. Autophagy 7: 707-715, 2011.

26. Liao A, Hu R, Zhao Q, Li J, Li Y, Yao K, Zhang R, Wang H, Yang W and Liu Z: Autophagy induced by FTY720 promotes apoptosis in U266 cells. Eur J Pharm Sci 45: 600-605, 2012.

27. Paglin S, Hollister T, Delohery T, Hackett N, McMahill M, Sphicas E, Domingo D and Yahalom J: A novel response of cancer cells to radiation involves autophagy and formation of acidic vesicles. Cancer Res 61: 439-444, 2001.

28. Kanzawa T, Germano IM, Komata T, Ito H, Kondo Y and Kondo S: Role of autophagy in temozolomide-induced cytotoxicity for malignant glioma cells. Cell Death Differ 11: 448-457, 2004.

29. Sato K, Tsuchihara K, Fujii S, Sugiyama M, Goya T, Atomi Y, Ueno T, Ochiai A and Esumi H: Autophagy is activated in colorectal cancer cells and contributes to the tolerance to nutrient deprivation. Cancer Res 67: 9677-9684, 2007.

30. Amaravadi RK, Yu D, Lum JJ, Bui T, Christophorou MA, Evan GI, Thomas-Tikhonenko A and Thompson CB: Autophagy inhibition enhances therapy-induced apoptosis in a Myc-induced model of lymphoma. J Clin Invest 117: 326-336, 2007.

31. Apel A, Herr I, Schwarz H, Rodemann HP and Mayer A: Blocked autophagy sensitizes resistant carcinoma cells to radiation therapy. Cancer Res 68: 1485-1494, 2008.

32. Bellodi C, Lidonnici MR, Hamilton A, Helgason GV, Soliera AR, Ronchetti M, Galavotti S, Young KW, Selmi T, Yacobi R, Van Etten RA, Donato N, Hunter A, Dinsdale D, Tirrò E, Vigneri P, Nicotera P, Dyer MJ, Holyoake T, Salomoni P and Calabretta B: Targeting autophagy potentiates tyrosine kinase inhibitor-induced cell death in Philadelphia chromosomepositive cells, including primary CML stem cells. J Clin Invest 119: 1109-1123, 2009. 